\title{
Angular "Butterfly" Fourier Filter for Suppression of Ray Artifacts in Tomographic Reconstructions
}

\section{Kováčik}

University in Prague, First Faculty of Medicine, Institute of Cellular Biology and Pathology, and Department of Cell Biology, Institute of Physiology, Academy of Sciences of the Czech Republic, v.v.i., Albertov 4, 12801 Prague 2, Czech Republic

Electron tomographic reconstructions suffer from a number of artifacts caused by effects accompanying the processes of acquisition of a set of tilted projections and its subsequent computational handling. The most pronounced artifacts usually come from imprecise projection alignment (or distortion of projections) and the presence of a region of missing data in the Fourier space, the "missing wedge". While there are commonly used methods that increase the precision of projection alignment [1-2], the artifacts of the missing wedge, the prolongation of reconstructed features in the z-axis and the presence of ray artifacts, often remain untreated.

The region of the missing wedge is in the Fourier space of the reconstructed object sharply delimited by planes of both highest-tilt projections (Fig. 1(a)). There is always a substantial step between values of Fourier transform in the planes of the two highest-tilt projections and in the zero-filled missing wedge. Therefore, the impulse response of the missing wedge contains two rays perpendicular to each of the two projections, which can be easily observed in planes perpendicular to the tilting axis (Figs. 1(c), 2). These rays emanate from each point of the reconstructed volume and often span its substantial portion, interfering thus with distant reconstructed structures or even creating false structural features. However, the rays can be suppressed by a simple Fourier filter whose design is presented in Fig. 1(b). Its purpose is (i) to attenuate the transition between the data and the missing wedge regions by multiplication of the Fourier transform values in data region points close to the highest-tilt planes by a gradual ramp oriented in the direction perpendicular to the highest-tilt plane (e.g. in form of the known low-pass Butterworth filter), and (ii) leave the Fourier values close to the tilting axis unchanged to prevent high-pass filter effects by overlaying the closeto-tilting axis values of the filter by another two-sided Butterworth ramp (Fig. 1(b)). The strength of the filter can be arbitrarily modified by changing the form of the ramps.

If all Fourier planes perpendicular to the tilting axis are multiplied by such filter, the rays are substantially eliminated (Figs. 2, 3). Moreover, the EFOMs of reconstructed noise-free test volumes before and after the angular filtering differed only insignificantly. Therefore, the presented angular filtering may be useful in both in qualitative and quantitative assessment of electron tomographic reconstructions, as can be observed in Figs. 2, 3.

\section{References}

[1] D. Mastronarde, Fiducial Marker and Hybrid Alignment Methods for Single-and Double-axis Tomography, in: J. Frank (ed.), Electron Tomography, Springer, New York, 2006, 163-186.

[2] L. Kovacik et al., J. Struct. Biol. 166 (2009) 263-271.

[3] This research was supported by the grants from the Ministry of Education, Youth and Sports of the Czech Republic MSM0021620806 and LC535, and by the grant of the Academy of Sciences of the Czech Republic AV0Z50110509. 


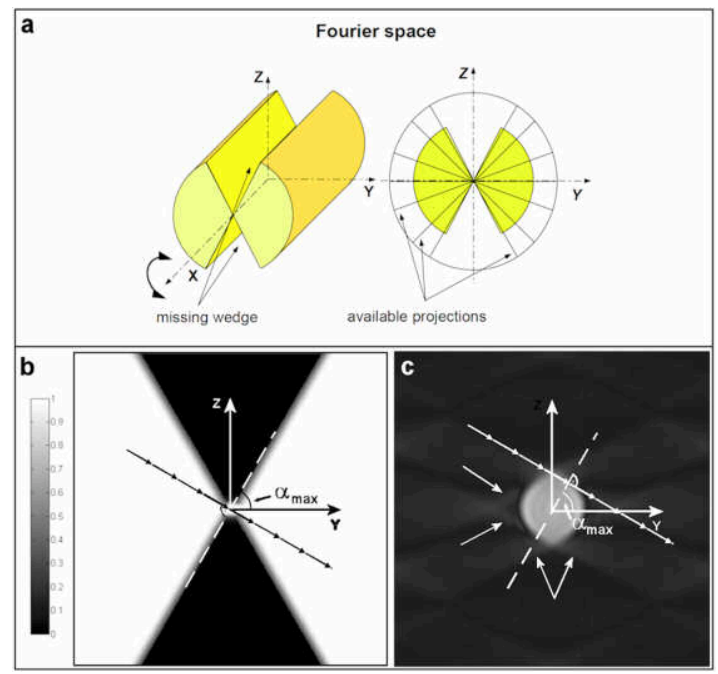

FIG. 1.: (a) Geometry of data and missing wedge region in Fourier space at single axis tilting. Projection data are available within yellow regions.

(b) Intensity plot of the angular filter. The filter dampens both sharp edges of the data region created by the highest tilt projections (dashed line), and keeps the frequencies in the central region close to the tilting axis unattenuated.

(c) In a cross-section perpendicular to the tilting axis through a noise-free reconstruction of a test cylinder, rays caused by the missing wedge run perpendicularly to the highest tilt projection (dashed-arrowed line, arrows).

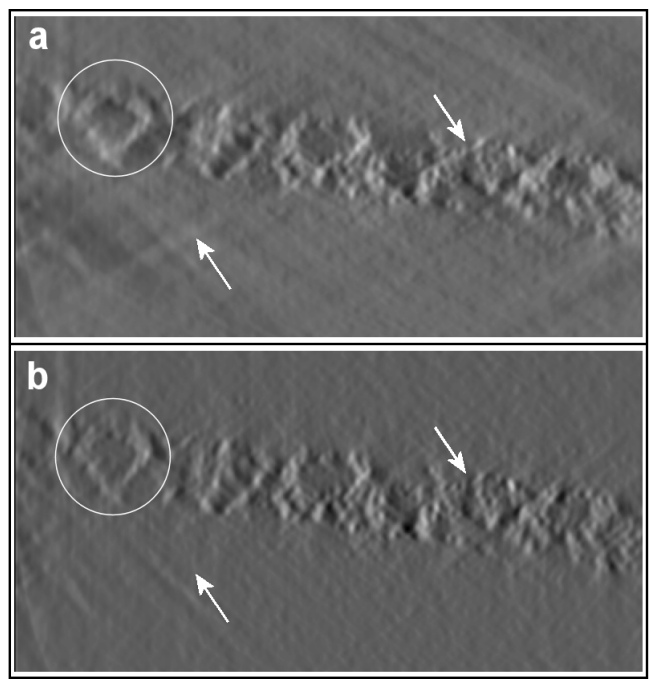

FIG. 2: Effect of the angular filtering in a cross-section perpendicular to the tilting axis through a 3D reconstruction of chemically fixed and contrasted lipid vesicles in rat's brain. Vesicles are encircled. Arrows indicate positions of rays perpendicular to the highest tilting angles.

(a) The Y-Z cross-section as required by bilinear interpolation in Fourier space.

(b) The same cross-section reconstructed from the interpolated data multiplicated in Fourier space with the angular filter.

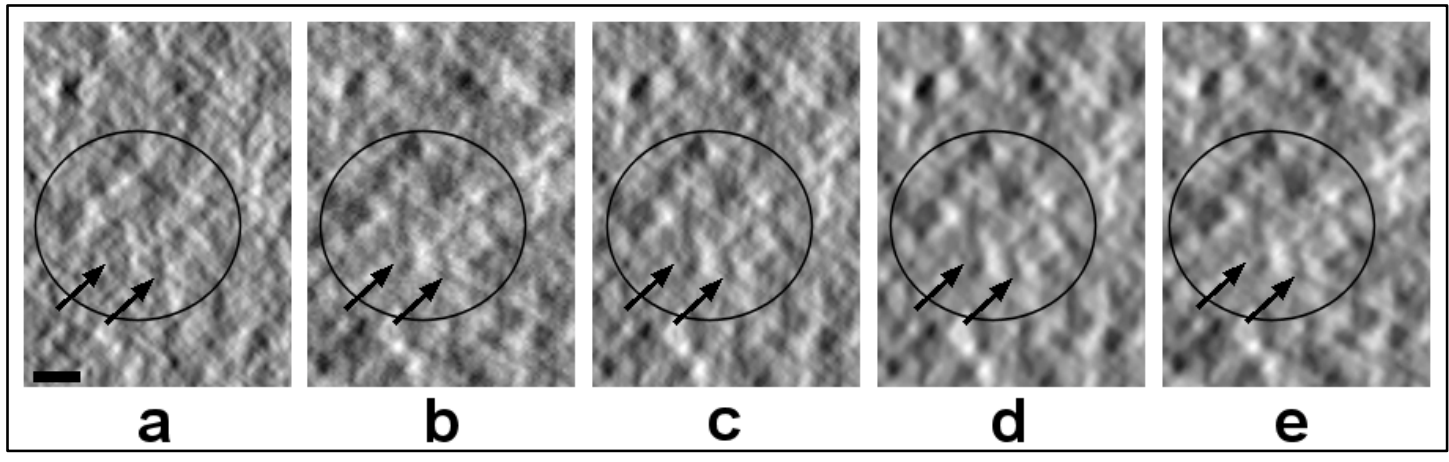

FIG.4: Cross-sections (perpendicular to tilting axis) through low-dose cryo-tomographic reconstructions of $30 \mathrm{~nm}$ chromatin fibers in low salt conditions, isolated from chicken erythrocytes, after 25 SIRT iterations (encircled). Reconstruction (a) was computed from CTF uncorrected projections and was angular filtered, volumes in (b)-(e) from were obtained form CTF corrected projections. Reconstructions were (b): angular unfiltered, (c): angular filtered, (d): angular and NAD (non-linear anisotropic diffusion) filtered, (e) NAD filtered. Arrows indicate positions where the rays caused by the missing wedge in (c) and (e) interfere with the reconstructed nucleosome structure, which can be more clearly identified in the angular filtered recontructions (c) and (d). 